\title{
IL-21 is required for CD4 memory formation in response to viral infection
}

\author{
Yuqing Yuan, ${ }^{1}$ Yiping Yang ${ }^{1,2}$ and Xiaopei Huang ${ }^{1}$ \\ 'Division of Hematologic Malignancies and Cellular Therapy, Department of Medicine, and ²Department of Immunology, \\ Duke University Medical Center, Durham, North Carolina, USA.
}

IL-21 has been shown to play an important role in the CD8 T cell response during acute and chronic viral infections. However, the role of IL-21 signaling in the CD4 T cell response to viral infection remains incompletely defined. In a model of infection with vaccinia virus, we show that intrinsic IL-21 signaling on CD4 T cells was critical for the formation of memory CD4 T cells in vivo. We further reveal that IL-21 promoted CD4 T cell survival in a mechanism dependent on activation of the STAT1 and STAT3 signaling pathways. In addition, the activation of Akt is also required for IL-21-dependent survival of CD4 T cells in vivo. These results identify a critical role for intrinsic IL-21 signaling in CD4 T cell survival and memory formation in response to viral infection in vivo and may provide insights into the design of effective vaccine strategies.

Conflict of interest: The authors have declared that no conflict of interest exists.

Submitted: September 9, 2016 Accepted: February 16, 2017 Published: April 6, 2017

Reference information: JCI Insight. 2017;2(7):e90652. https:// doi.org/10.1172/ji.insight.90652.

\section{Introduction}

IL-21, which is produced mainly by activated CD4 T cells (1) and NKT cells (2), is a member of the common- $\gamma$ chain family of cytokines that includes IL-2, IL-4, IL-7, IL-9, and IL-15 (1, 3). Previous studies have shown that IL-21 plays an important role in the regulation of both innate and adaptive immune responses (3). IL-21 is critical for NK cell activation and expansion $(1,4)$. IL-21 also promotes terminal B cell differentiation into plasma cells and antibody production (5, 6). Furthermore, IL-21 enhances antigen-specific CD8 T cell expansion (7) and the differentiation of follicular helper T cells in vivo (8). IL-21 also promotes the differentiation of the inflammatory Th17 lineage $(9,10)$ and contributes to autoimmunity $(3)$. In addition, it has been shown that direct action of IL-21 on CD8 T cells is required to sustain the CD8 T cell response during chronic viral infections (11-13).

Recent studies have shown that IL-21 signaling is also critical for the formation of memory CD8 T cells in response to acute viral infections (14-16). This is achieved by promoting the survival of effector CD8 $\mathrm{T}$ cells in a STAT1- and STAT3-dependent manner (14). Although IL-21 is mainly produced by activated CD4 T cells (1), it remains to be determined whether IL-21 signaling is also required for the formation of memory CD4 T cells following an acute viral infection.

In this study, we show that direct IL-21 signaling on CD4 $\mathrm{T}$ cells was also required for the formation of memory CD4 T cells in response to infection with vaccinia virus (VV) in vivo. We further found that the activation, proliferation, or effector differentiation of CD4 T cells in response to VV infection in vivo was not affected by lack of IL-21 signaling. However, the survival of activated CD4 T cells was critically dependent on direct IL-21 signaling on CD4 T cells. In addition, we found in vitro that IL-21 signaling promoted CD4 T cell survival by activating the STAT1 and STAT3 signaling pathways. In vivo, CD4 T cells defective for IL-21 signaling had reduced levels of STAT1 and STAT3 activation in response to VV infection. We further demonstrated that the activation of Akt in CD4 T cells was compromised in the absence of IL-21 signaling and that the IL-21/Akt pathway was critical for CD4 T cell survival in vivo. Altogether, our study indicates that direct IL-21 signaling is required for the survival of activated CD4 T cells and the formation of long-lived memory cells in response to VV infection.

\section{Results}

Intrinsic IL-21 signaling is required for CD4 memory formation in vivo. To study whether IL-21 signaling is required for the formation of memory CD4 $\mathrm{T}$ cells in response to $\mathrm{VV}$ infection, naive WT or IL-21R $\mathrm{R}^{-/}$influenza HA-specific CD4 T cells (Thy1.2+) derived from 6.5 TCR-HA transgenic mice were transferred into congenic Thy $1.1^{+}$mice that were subsequently infected with recombinant VV encoding HA (VV-HA). At different time points after infection, splenocytes were analyzed for the presence of the clonotypic CD4 T cells. Seven days 
A
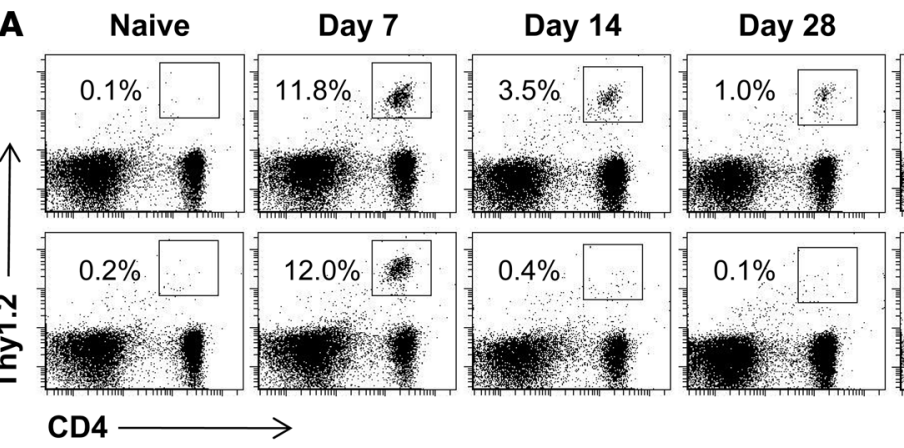

Day 42

Day $42+5$

B

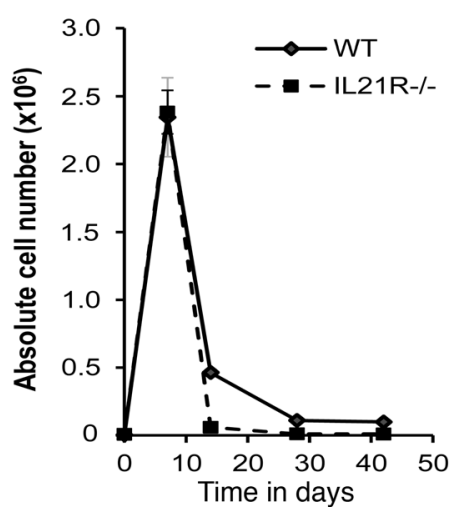

C

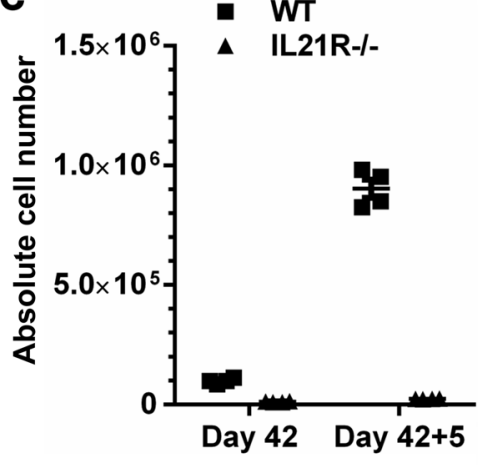

Figure 1. Lack of IL-21 signaling in HA-specific CD4 T cells leads to defective CD4 memory formation in the response to VV infection. $5 \times 10^{4}$ naive clonotypic HA-specific CD4 T cells (Thy1.2+) sorted from WT or IL-21R ${ }^{-/-}$TCR-HA transgenic mice were transferred into Thy1.1+ mice intravenously, followed by infection with $5 \times 10^{6}$ pfu VV-HA i.p., or left uninfected as naive controls. 7, 14, 28, and 42 days after infection, splenocytes were harvested and stained with anti-CD4 and anti-Thy1.2. (A) FACS plots with percentages of total clonotypic CD4 T cells among total CD4 T cells. (B) Mean absolute numbers \pm SEM of clonotypic CD4 T cells per spleen ( $n=4$ per group). (C) At day 42 , some mice were rechallenged with Ad-HA $\left(2 \times 10^{9} \mathrm{pfu}\right.$ ) intravenously and harvested 5 days later (day $\left.42+5\right)$ for recall responses. Mean absolute numbers \pm SEM of clonotypic CD4 T cells per spleen before (day 42) and after (day $42+5$ ) rechallenge with Ad-HA are indicated ( $n$ $=4$ per group). Data are representative of 3 independent experiments.

after infection, a similar degree of clonal expansion was observed for both the WT and IL-21R ${ }^{-/-}$HA-specific CD4 T cells (Figure 1, A, and B). Thereafter, WT HA-specific CD4 T cells underwent contraction to form a memory pool (Figure 1, A and B), whereas IL-21R ${ }^{-/}$HA-specific CD4 T cells could not survive the contraction phase to develop into memory cells (Figure 1, A and B). To ensure that the lack of memory formation with IL-21R $\mathrm{R}^{-/}$HA-specific $\mathrm{T}$ cells was not due to a low sensitivity of detection in our system, we boosted mice at day 42 with recombinant adenovirus encoding HA (Ad-HA) to assess the recall response. We observed a robust recall expansion of WT HA-specific memory CD4 T cells (Figure 1C). However, there were still no detectable IL-21R $\mathrm{R}^{-/-} \mathrm{HA}$-specific CD4 T cells (Figure 1C).

To further test that the importance of IL-21 in the CD4 T cell memory response to viral infection is not just restricted to a particular $\mathrm{T}$ cell receptor (TCR), we repeated the experiments with OVA-specific CD4 T cells derived from DO.11 TCR-OVA transgenic mice. Indeed, we showed that, similar to HA-specific T cells (Figure 1, A and B), IL-21R - $^{-}$OVA-specific CD4 T cells could not develop into memory cells (Figure 2, A and B), suggesting that the role of IL-21 in CD4 memory formation is not restricted to a single TCR. However, in contrast to HA-specific T cells, we found that IL-21 deficiency in OVA transgenic $\mathrm{T}$ cells led to a reduction in clonal expansion at day 7 (Figure 2A). To further confirm this observation, WT (Thy $1.2^{+}$) and IL-21R $\mathrm{R}^{-/-}$(Thy $1.1^{+}$) OVA-specific CD4 $\mathrm{T}$ cells were mixed at a 1:1 ratio and cotransferred into congenic mice that were subsequently infected with VV encoding OVA (VV-OVA). Seven and twenty-one days after infection, splenocytes were

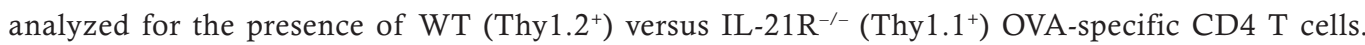

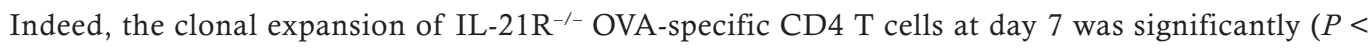
0.05 ) reduced compared with the WT counterparts, leading to no detectable memory cells by day 21 (Figure 2, C and D). The reason for the differential clonal expansion between two different clo- 
A

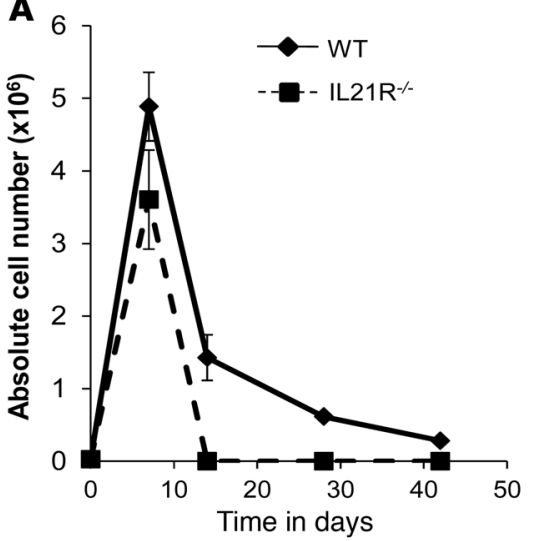

B

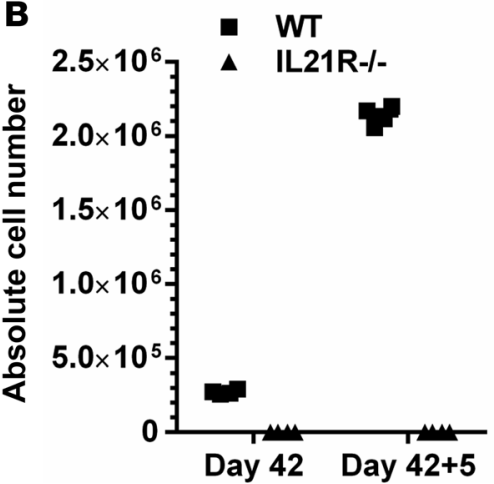

C
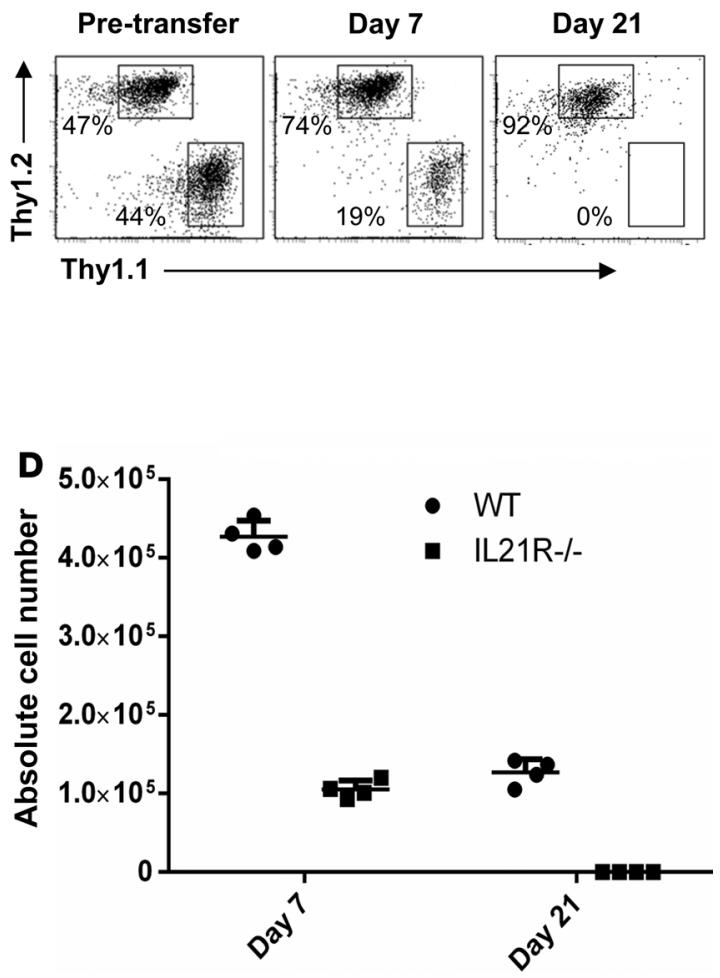

Figure 2. IL-21-deficient OVA-specific CD4 T cells fail to develop memory response to VV infection. (A and B) $1 \times 10^{5}$

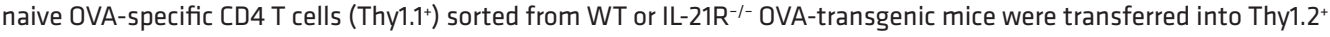
mice intravenously, followed by infection with $5 \times 10^{6}$ pfu VV-OVA i.p., or were left uninfected as naive controls. 7, 14,28 , and 42 days after the infection, splenocytes were stained with anti-CD4 and anti-Thy1.1. (A) Mean absolute numbers \pm SEM of clonotypic CD4 T cells per spleen ( $n=4$ per group). At day 42 , some mice were rechallenged with Ad-OVA $\left(2 \times 10^{9} \mathrm{pfu}\right)$ intravenously and harvested 5 days later (day $\left.42+5\right)$ for recall responses. (B) Mean absolute numbers \pm SEM of clonotypic CD4 T cells per spleen before (day 42) and after (day 42+5) rechallenge with Ad-OVA ( $n=$

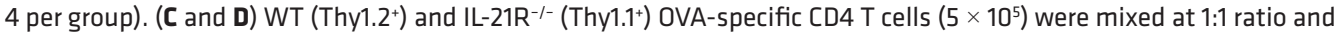
cotransferred into Thy $1.1^{+}$congenic mice, followed by infection with $5 \times 10^{6}$ pfu VV-OVA. 7 and 21 days after infection, splenocytes were stained with anti-CD4, anti-Thy1.1, anti-Thy1.2, and anti-D0.11 OVA-TCR (KJ1-26) and analyzed for

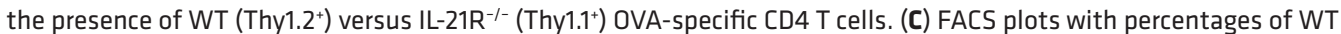

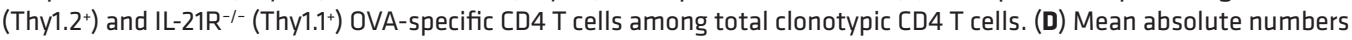

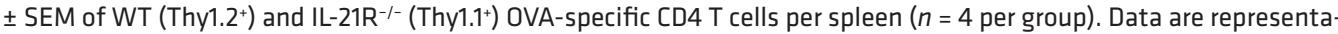
tive of 3 independent experiments.

notypic $\mathrm{T}$ cells remains unclear but could be related to reduced proliferation and/or increased apoptosis in OVA-specific CD4 T cells compared with the HA-specific T cells. Nevertheless, these data further support our observation that intrinsic IL-21 signaling in CD4 T cells is critical for the formation of long-lived memory cells in response to VV infection.

IL-21 signaling is critical for the survival of activated CD4 T cells in vivo. Lack of CD4 memory formation in the absence of intrinsic IL-21 signaling could be due to insufficient activation, proliferation, or effector differentiation or poor survival. To address these possibilities, we transferred naive WT or IL-21R ${ }^{-1-}$ HA-specific CD4 T cells (Thy $1.2^{+}$) into congenic Thy $1.1^{+}$mice and subsequently infected the hosts with VV-HA. Twenty-four hours after infection, both WT and IL-21R ${ }^{-/-}$clonotypic CD4 T cells displayed a similarly activated $\mathrm{CD} 44^{\text {hi }} \mathrm{CD} 69^{\text {hi }}$ phenotype compared with the naive $\mathrm{CD} 44^{\text {lo }} \mathrm{CD} 69^{\text {lo }}$ phenotype (Figure 3 , A and $\mathrm{B}$ ), suggesting that the activation of $\mathrm{CD} 4 \mathrm{~T}$ cells early during infection is not affected by lack of IL-21 signaling. Three days after infection, CFSE-labeled WT and IL-21R ${ }^{-/-}$clonotypic CD4 T cells underwent similar levels of proliferation, as measured by CFSE dilution (Figure 3C), suggesting that lack of IL-21 signaling does not affect CD4 T cell proliferation in vivo. Furthermore, IL-21R $\mathrm{R}^{-/}$clonotypic CD4 T cells produced similar levels of IFN- $\gamma(P>0.05)$, further confirming that activation of clonotypic CD4 T cells is not affected in the absence of IL-21 signaling (Figure 3, D and E). 


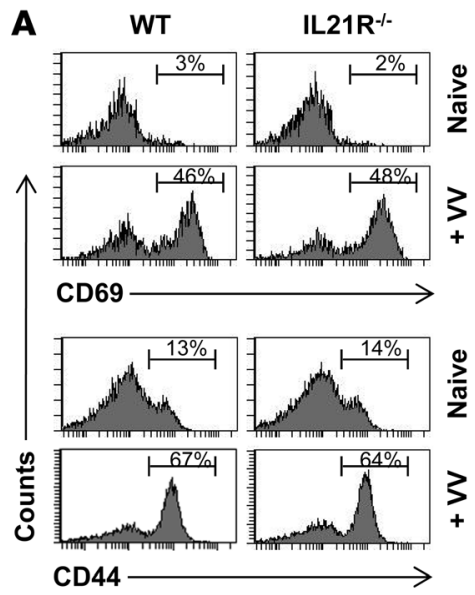

C

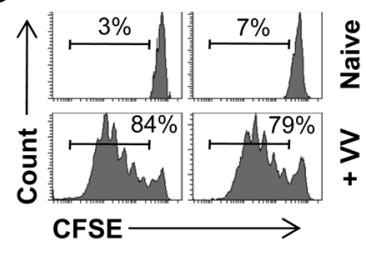

D WT IL21R ${ }^{-1}$
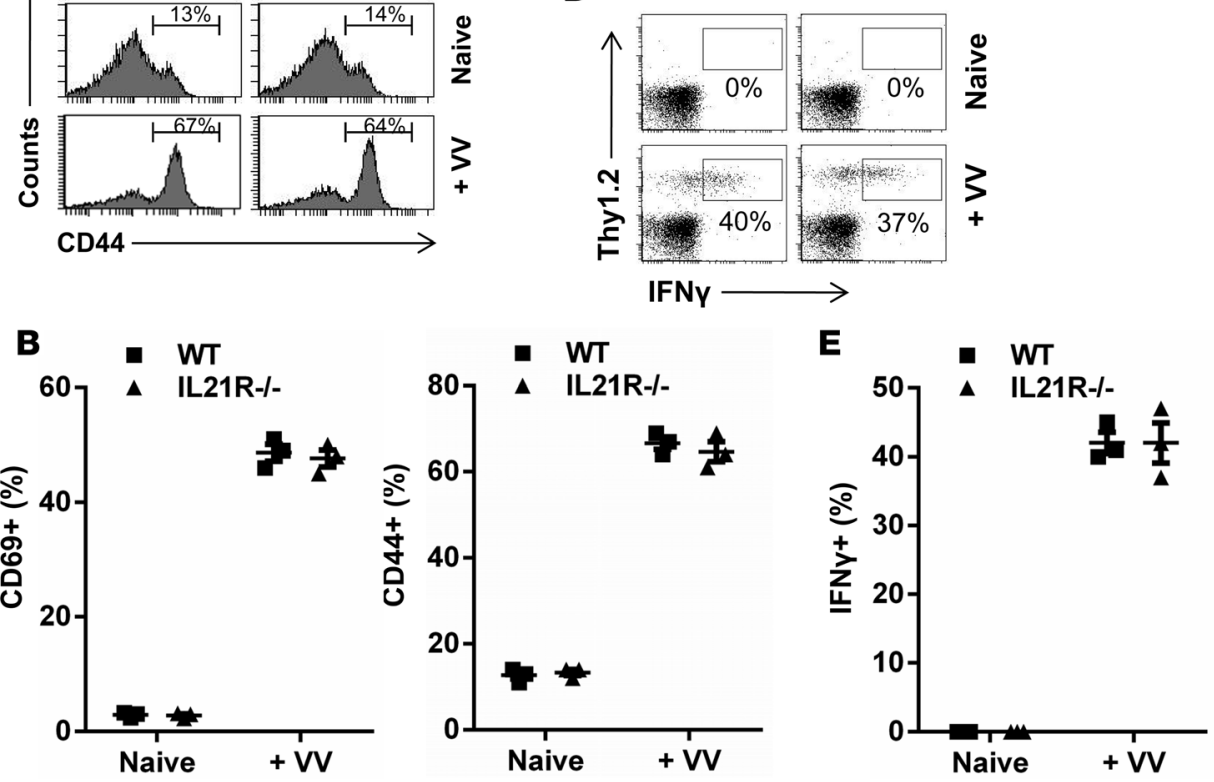

Figure 3. The activation of CD4 T cells is independent of IL-21 signaling. $5 \times 10^{5}$ purified naive clonotypic CD4 T cells from WT or IL-21R ${ }^{-1-}$ TCR-HA transgenic mice (Thy1.2+) were transferred into Thy1.1+ congenic recipients that were subsequently infected with VV-HA $\left(5 \times 10^{6} \mathrm{pfu}\right.$, i.p., +VV). Some mice were left uninfected (Naive). (A and B) Twenty-four hours after infection, splenocytes were harvested and stained with antibodies to CD4, Thy1.2, and the activation markers CD44 or CD69. (A) FACS plots and the percentages of CD44 ${ }^{\text {hi }}$ and CD69 ${ }^{\text {hi }}$. (B) Mean absolute numbers \pm SEM of CD44 ${ }^{\text {hi }}$ and CD69 $^{\text {hi }}$ ( $n=4$ per group). (C) Three days after infection, in vivo division of CFSE-labeled clonotypic cells in the spleen was analyzed, and the percentages of CFSE-positive cells are shown. ( $\mathbf{D}$ and $\mathbf{E}$ ) Seven days after infection, splenocytes were stained with anti-CD4, anti-Thy1.2, and anti-IFN- $\gamma$ intracellularly. (D) FACS plots and the percentages of IFN- $\gamma$-producing clonotypic cells. (E) Mean absolute numbers \pm SEM of IFN- $\gamma$-producing clonotypic cells among clonotypic CD4 T cells ( $n=4$ per group). Data shown are representative of 3 independent experiments.

We next determined whether the lack of memory formation in IL-21R ${ }^{-/-}$CD4 $\mathrm{T}$ cells was due to enhanced apoptosis in the absence of IL-21 signaling. We transferred naive WT or IL-21R ${ }^{-1-}$ HA-specific CD4 T cells into naive hosts that were subsequently infected with VV-HA. Seven days later, splenocytes were stained with annexin $\mathrm{V}$ to detect cells undergoing apoptosis. Indeed, IL-21R $\mathrm{R}^{-/}$clonotypic CD4 $\mathrm{T}$ cells had a significant $(P<0.05)$ increase in annexin $\mathrm{V}$ compared with WT clonotypic cells (Figure $4, \mathrm{~A}$ and $\mathrm{B})$. This is correlated with a significant $(P<0.05)$ reduction in the expression of the prosurvival factor, Bcl-xL, in IL-21 $\mathrm{R}^{-/-}$clonotypic CD4 $\mathrm{T}$ cells compared with the WT control (Figure 4, C and D). These results suggest that the survival of activated CD4 T cells is critically dependent on intrinsic IL-21 signaling in vivo.

IL-21-dependent survival of activated CD4 T cells is mediated by STAT1 and STAT3. We next sought to investigate how intrinsic IL-21 signaling promotes the survival of CD4 T cells. It has been shown that IL-21-dependent CD8 T cell survival is mediated by the STAT1 and STAT3 pathways (14). To study whether these STAT pathways also play a role in IL-21-mediated CD4 T cell survival, we first cultured naive polyclonal CD4 T cells in the presence of recombinant IL-21 and assessed the activation of STAT1 and STAT3 by intracellular staining for phosphorylated STAT1 (pSTAT1) and phosphorylated STAT3 (pSTAT3). Increased activation of both STAT1 and STAT3 was observed in cultures supplemented with IL-21, compared with the medium-only control $(P<0.05)$ (Figure 5, A and B). 


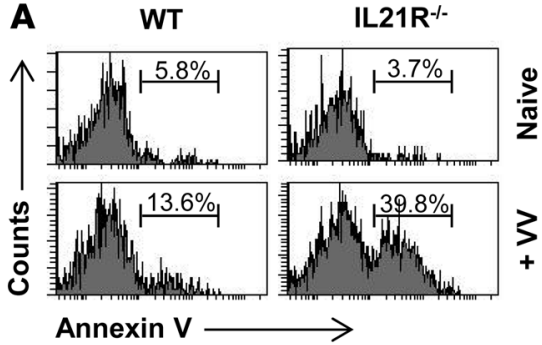

C

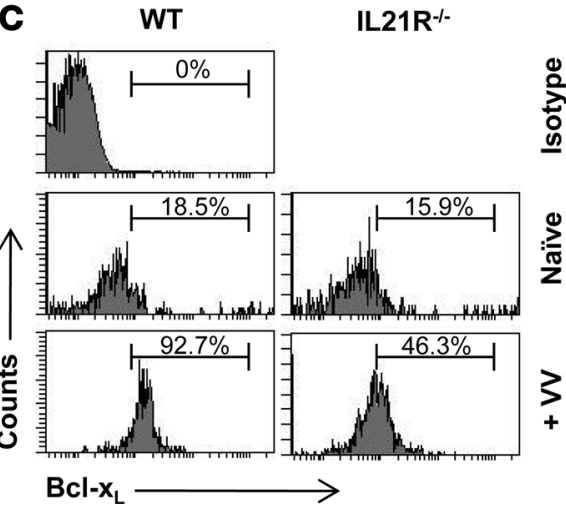

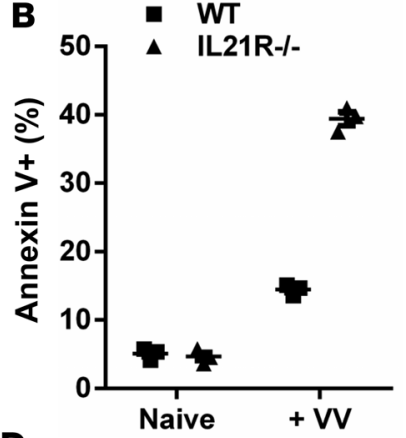

D

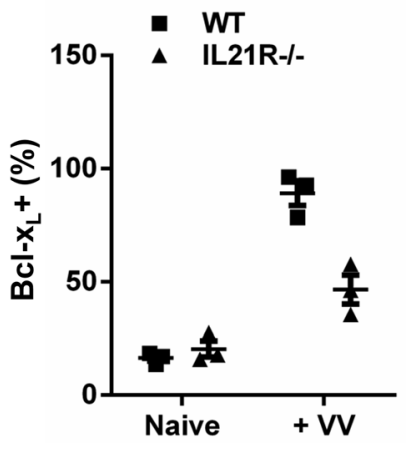

Figure 4. IL-21 signaling is required for the survival of activated CD4 T cells. $5 \times 10^{5}$ purified naive clonotypic CD4 T cells from WT or IL-21R ${ }^{-1-}$ TCR-HA transgenic mice (Thy $1.2^{+}$) were transferred into Thy $1.1^{+}$congenic recipients that were subsequently infected with $\mathrm{VV}-\mathrm{HA}$ $(+V V)$. Some mice were left uninfected (Naive). Seven days after infection, splenocytes were stained with (A) anti-CD4, anti-Thy1.2, and annexin $\mathrm{V}$ or (C) Bcl- $\mathrm{X}_{\perp}$. The mean percentages \pm SEM of (B) annexin $\mathrm{V}^{+}$and $(\mathbf{D}) \mathrm{BCl}-$ $\mathrm{X}_{\mathrm{L}}{ }^{+}$clonotypic CD4 T cells are indicated ( $n=4$ per group). All plots are gated on CD4 ${ }^{+}$Thy $1.2^{+}$cells. Data shown are representative of 3 independent experiments.

We then examined whether IL-21 signaling on CD4 T cells promotes activation of STAT1 and STAT3 upon VV infection in vivo. Naive WT or IL-21R ${ }^{-/}$HA-specific CD4 T cells were transferred into congenic mice that were subsequently infected with VV-HA. Five days after infection, splenocytes were harvested and stained intracellularly for pSTAT1 and pSTAT3. Significant $(P<$ $0.05)$ activation of both STAT1 and STAT3 was observed in WT clonotypic CD4 T cells upon VV infection in vivo, compared with the naive control (Figure 5, C and D). However, the extent of

STAT1 and STAT3 activation was significantly $(P<0.05)$ reduced in IL-21R ${ }^{-/}$clonotypic CD4 T cells, compared with their WT counterparts (Figure 5, C and D). This reduction was not due to a decreased level of total STAT1 or STAT3 in IL-21R ${ }^{-/-}$cells (Figure 5, C and D). These results indicate that intrinsic IL-21 signaling in CD4 T cells is required for efficient activation of STAT1 and STAT3.

To further determine whether the STAT1 and STAT3 pathways were required for IL-21-dependent CD4 T cell survival, naive WT or STAT1 $1^{-1-}$ polyclonal CD4 T cells were retrovirally transduced with either empty vector or a STAT3 dominant-negative (STAT3-DN) vector and cultured in the presence of IL-21. We observed that CD4 T cell apoptosis was significantly $(P<0.05)$ reduced when WT cells with empty vector were cultured in the presence of IL-21 compared with WT cells with the medium alone (Figure 6). However, this inhibition of apoptosis by IL-21 was abrogated in the absence of STAT1 (Figure 6), suggesting that STAT1 is critical for IL-21-dependent CD4 T cell survival. In addition, WT cells transduced with STAT3-DN also showed a reduction in IL-21-induced inhibition of apoptosis (Figure 6). Collectively, these data suggest that IL-21-dependent CD4 T cell survival is mediated by both the STAT1 and STAT3 pathways.

The activation of Akt is also required for IL-21-dependent CD4 T cell survival. In addition to the STAT1 and STAT3 pathways, the PI3K/Akt pathway has also been implicated in promoting IL-21-mediated $\mathrm{T}$ cell survival in vitro (17). To test whether the PI3K/Akt pathway is also important in promoting CD4 T cell survival in response to VV infection in vivo, we compared the activation of Akt in WT versus IL-21R ${ }^{-/-}$HA-specific CD4 $\mathrm{T}$ cells upon VV infection. Naive WT or IL-21R ${ }^{-/}$clonotypic CD4 T cells were transferred into congenic mice that were subsequently infected with VV-HA. Five days after infection, splenocytes were harvested and stained intracellularly for pAkt. Marked activation of Akt was observed in WT clonotypic CD4 T cells, compared with the naive control (Figure 7). However, the extent of Akt activation was significantly $(P<0.05)$ reduced in IL-21R ${ }^{-/-}$clonotypic CD4 T cells, compared with their WT counterparts (Figure 7), suggesting that intrinsic IL-21 signaling in CD4 T cells is also important for efficient activation of Akt.

To further examine the role of the Akt pathway in IL-21-mediated CD4 T cell survival in vivo, we first tested whether WT clonotypic CD4 T cells transduced with an Akt dominant-negative retrovirus (DN Akt) had a reduced survival upon VV infection. Seven and fifteen days after infection, a significant reduction in the percentage as well as the absolute cell numbers of the DN Akt-transduced CD4 $\mathrm{T}$ cells was observed, compared with the empty vector-transduced WT cells $(P<0.05)$ (Figure 8$)$. We 
A

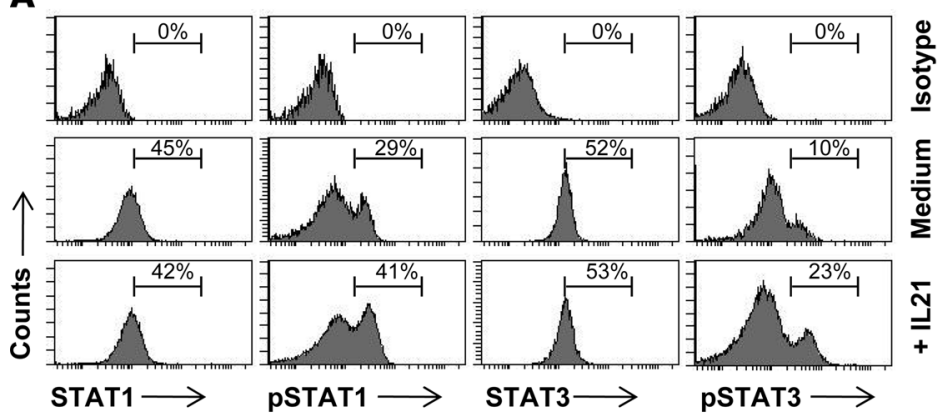

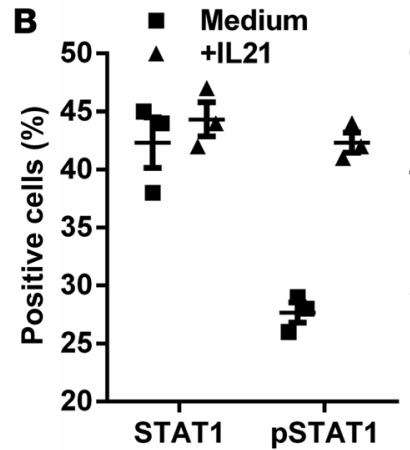

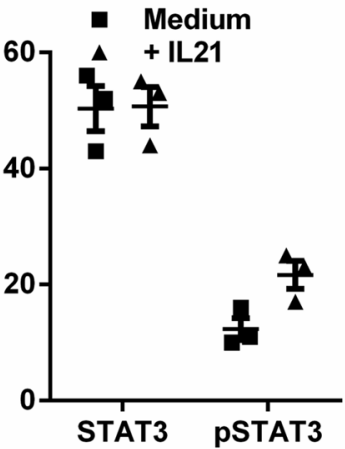

C

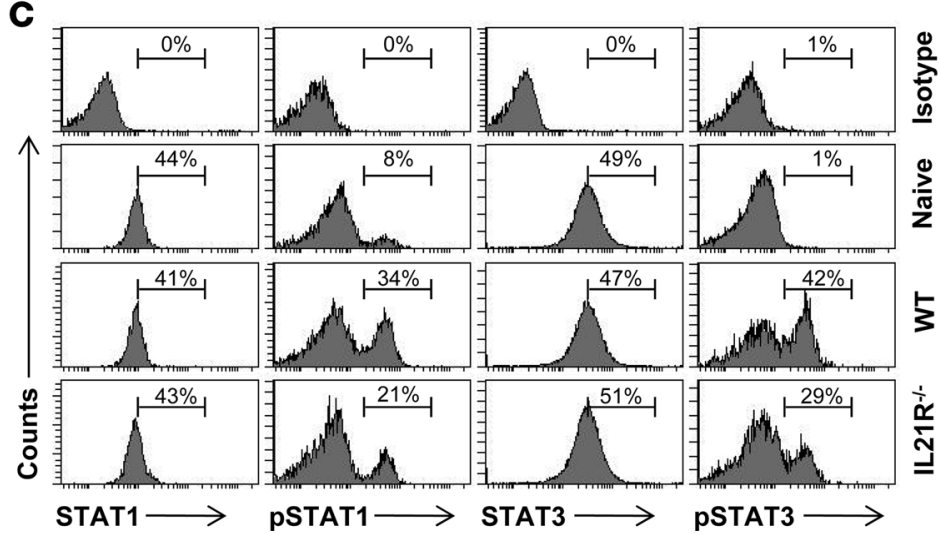

D

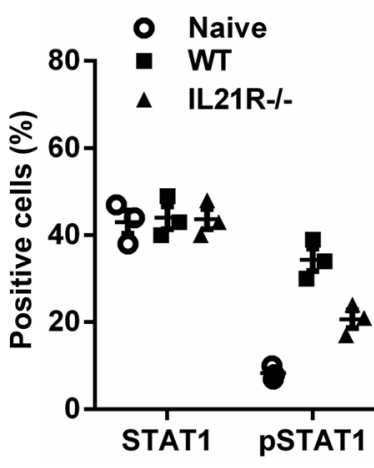

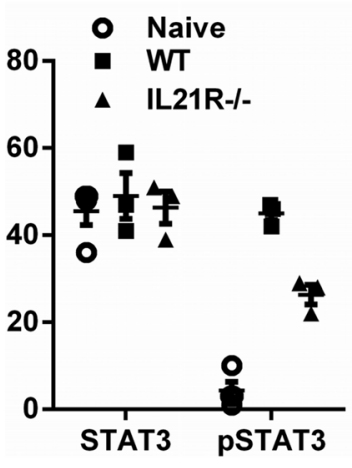

Figure 5. IL-21 signaling promotes activation of STAT1 and STAT3. (A and B) Purified polyclonal CD4 T cells were cultured for 1 hour in medium alone (Medium) or medium supplemented with recombinant murine IL-21 (+ IL21). (A) Cells were stained with anti-STAT1, anti-pSTAT1, anti-STAT3, or antipSTAT3 intracellularly. (B) Mean percentages \pm SEM of STAT1-, pSTAT1-, STAT3-, or pSTAT3-positive cells $\left(n=4\right.$ per group). (C and D) $5 \times 10^{5}$ purified naive clonotypic CD4 T cells from WT or IL-21R ${ }^{-1-}$ TCR-HA transgenic mice (Thy1.2+) were transferred to Thy1.1+ congenic recipients that were subsequently infected with VV-HA $\left(5 \times 10^{6} \mathrm{pfu}\right)$. Some mice were left uninfected (Naive). (C) Seven days after infection, splenocytes were stained with anti-CD4 and anti-Thy1.2 as well as anti-pSTAT1, anti-pSTAT3, anti-STAT1, anti-STAT3, or an isotype control intracellularly. (D) Mean percentages \pm SEM of pSTAT1 ${ }^{+}$and pSTAT3 ${ }^{+}$among clonotypic CD4 T cells ( $n=4$ per group). Data are representative of 3 independent experiments.

then tested whether the reduced survival of IL-21 $\mathrm{R}^{-1-}$ clonotypic CD4 $\mathrm{T}$ cells upon VV infection could be rescued by transduction with a constitutive activated form of Akt (constitutive Akt). Indeed, the transduction of constitutive Akt promoted the survival of IL-21R ${ }^{-1-}$ CD4 T cells $(P<0.05)$ (Figure 8)

Taken together, these results suggest that the activation of Akt is also critical for IL-21-dependent CD4 $\mathrm{T}$ cell survival in response to $\mathrm{VV}$ infection in vivo.

\section{Discussion}

In this study, we demonstrate that IL-21 signaling on CD4 $\mathrm{T}$ cells is critical for the formation of memory $\mathrm{CD} 4 \mathrm{~T}$ cells in response to VV infection. This is achieved by promoting the survival of activated CD4 T cells in a STAT1- and STAT3-dependent manner. We further reveal that activation of Akt is also required for IL-21-dependent survival of CD4 T cells in vivo.

What is responsible for the generation and maintenance of the memory $\mathrm{T}$ cell population remains incompletely defined. Studies have shown that inflammatory signals (18) and the nature and the strength of TCR stimulation (19-21) as well as the costimulation (22) can promote the formation of memory $\mathrm{T}$ cells. In addition, the common- $\gamma$ chain cytokines IL-7 and IL-15 are also critical in the maintenance of memory $\mathrm{T}$ cells $(23,24)$. Although IL-21 has been shown to be important for the formation of memory CD8 T cells in response to acute viral infections (14-16), its role in CD4 T cell responses remains unclear. In a model of VV infection, here we provided evidence that IL-21 signaling is also required for the formation of memory $\mathrm{CD} 4 \mathrm{~T}$ cells following an acute viral infection in vivo. 


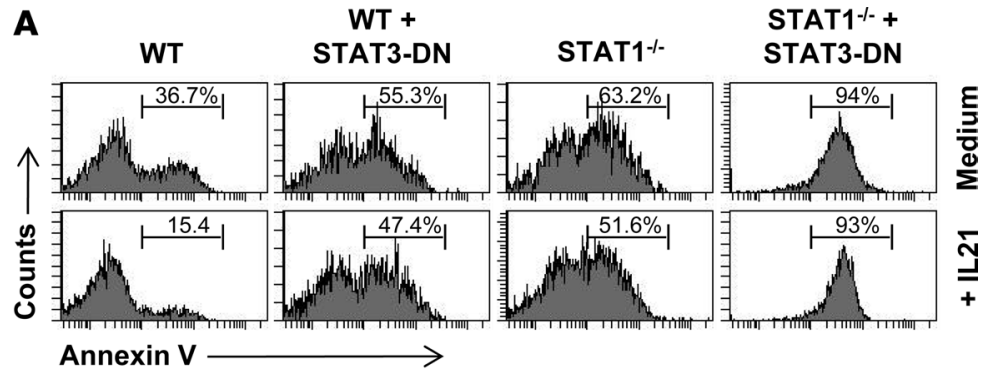

Figure 6. IL-21-mediated CD4 $\mathrm{T}$ cell survival is dependent on STAT1 and STAT3. Purified polyclonal CD4 ${ }^{+} \mathrm{T}$ cells from WT or STAT1 $^{-1-}$ mice were stimulated with PMA $(40 \mathrm{ng} / \mathrm{ml})$ and ionomy$\operatorname{cin}(500 \mathrm{ng} / \mathrm{ml})$ in the presence of IL-2 $(100 \mathrm{U} / \mathrm{ml})$ for 24 hours and then retrovirally infected with empty vector or dominant-negative STAT3 vector (STAT3-DN) by spin infection. (A) Treated cells cultured in medium alone (medium) or in medium supplemented with IL-21 (+ IL21) for 4 more days were stained with annexin V. (B) Mean percentages \pm SEM of annexin $\mathrm{V}^{+}$cells ( $n=4$ per group). Data shown are representative of 3 independent experiments.

Previous studies have shown that IL-21 signaling in CD8 $\mathrm{T}$ cells can preferentially activate the STAT1 and STAT3 pathways, leading to enhanced $\mathrm{T}$ cell proliferation in combination with IL-15 in vitro (25) and CD8 T cell survival and memory formation in vivo (26). Here, we show that IL-21 signaling also activates both STAT1 and STAT3 pathways in CD4 T cells in vitro and that IL-21 enhances the survival of anti-CD3-activated CD4 $\mathrm{T}$ cells in a STAT1- and STAT3-dependent manner. In addition to the STAT1 and STAT3 pathways, the PI3K/ Akt pathway has been shown to promote IL-21-mediat-

ed T cell survival in vitro (17). Indeed, we demonstrated in this study that the activation of Akt is also critical for IL-21-dependent CD4 T cell survival in response to VV infection in vivo. It remains to be determined in the future whether the STAT1/STAT3 and Akt pathways interact and coordinate to promote CD4 T cell survival.

In conclusion, we have shown that direct action of IL-21 on CD4 T cells is required for the formation of memory CD4 $\mathrm{T}$ cells in response to $\mathrm{VV}$ infection in vivo through promotion of the survival of activated CD4 T cells. This IL-21-dependent CD4 T cell survival is mediated through the STAT1 and STAT3 signaling pathways. In addition, the activation of Akt is also required for IL-21-dependent survival of CD4 T cells in vivo. These results identify a critical role for intrinsic IL-21 signaling in CD4 $\mathrm{T}$ cell survival and memory formation in response to viral infection in vivo and may provide insights into the design of effective vaccine strategies.

\section{Methods}

Mice. B10.D2 and 129/Sv mice were purchased from The Jackson Laboratory and Charles River Laboratories, respectively. IL-21 $\mathrm{R}^{-/}$mice on a BALB/c background were a gift from W.J. Leonard (NIH, Bethesda, Maryland, USA) (5) and were backcrossed to the B10.D2 background for 9 generations. $\mathrm{STAT}^{-/-}$mice on the 129/Sv background were purchased from Taconic. The 6.5 TCR-HA transgenic mice that express a TCR recognizing an I-E ${ }^{\mathrm{d}}$-restricted HA epitope $\left({ }^{110}\right.$ SFERFEIFPKE $\left.{ }^{120}\right)$ were pro-

Figure 7. IL-21 signaling also promotes activation of Akt in vivo. $1 \times 10^{6}$ purified naive clonotypic $\mathrm{HA}$-specific CD4 T cells from WT or IL-21R ${ }^{-1-}$ TCR-HA transgenic mice (Thy1.2+) were transferred into Thy $1.1^{+}$congenic recipients that were subsequently infected with VV-HA ( $\left.5 \times 10^{6} \mathrm{pfu}\right)$. (A) Some mice were left uninfected (Naive). Five days after infection, splenocytes were stained with anti-CD4 and anti-Thy1.2 as well as anti-Akt or anti-pAkt or an isotype control intracellularly. (B) Mean percentages $\pm \mathrm{SEM}$ of $\mathrm{Akt}^{+}$and $\mathrm{pAkt}^{+}$among clonotypic CD4 T cells ( $n=4$ per group). Data are representative of 3 independent experiments.
A

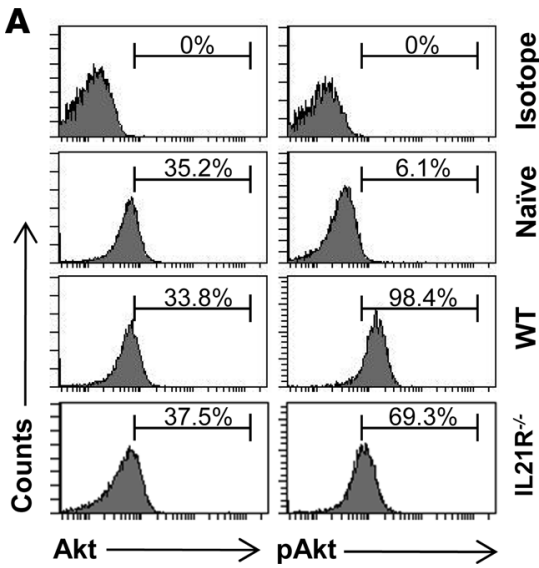

B

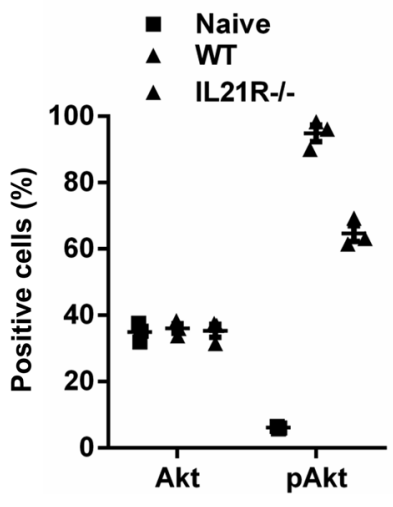


A

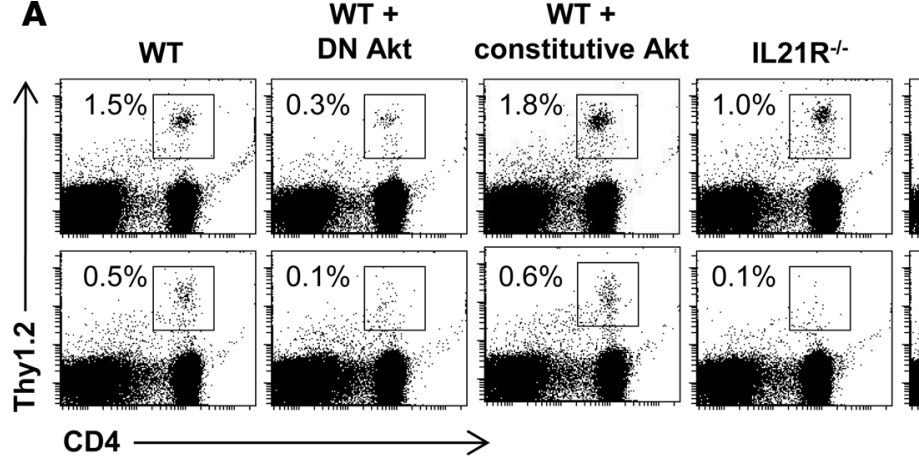

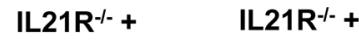

DN Akt constitutive Akt
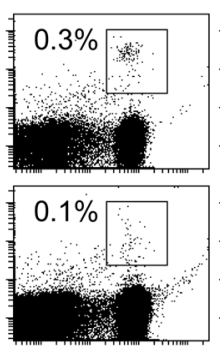
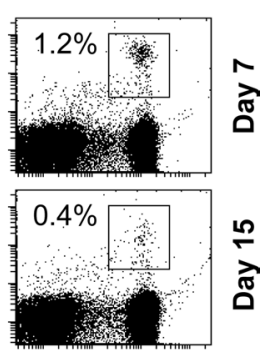

B

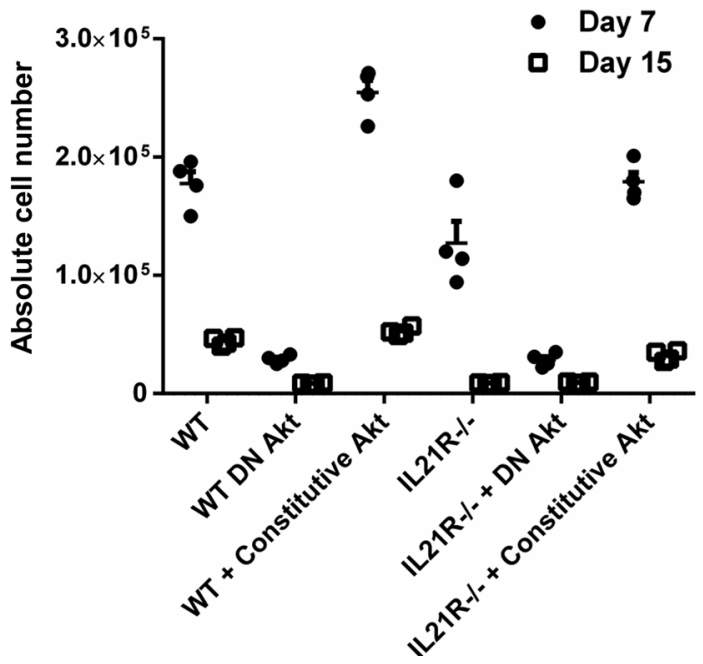

Figure 8. Alkt is required for IL-21-dependent CD4 T cell survival in vivo. Purified naive clonotypic HA-specific CD4 T cells from WT or IL-21R ${ }^{-1-}$ TCRHA transgenic mice were stimulated with $40 \mathrm{ng} / \mathrm{ml} \mathrm{PMA}$ and $500 \mathrm{ng} / \mathrm{ml}$ ionomycin for 24 hours in vitro. Stimulated cells were then infected with retrovirus-encoding dominant-negative (DN) Akt or constitutively activated (constitutive) Akt. The retroviral vectors carried GFP. Thus, the GFP+ cells after infection were selected by sorting. $5 \times 10^{4} \mathrm{GFP}^{+}$WT or IL21R-1- HA-specific T cells were transferred into Thy1.1 mice intravenously. Recipient mice were subsequently infected with $5 \times 10^{6} \mathrm{pfu}$ VV by i.p. injection. Splenocytes were harvested for analysis 7 and 15 days after infection. (A) The percentage of GFP+Thy1.2+ $2^{+}$D4 $4^{+}$cells among total $\mathrm{CD} 4^{+} \mathrm{T}$ cells. (B) The mean absolute numbers \pm SEM of GFP+Thy $1.2^{+} \mathrm{CD}^{+}$cells per spleen $(n=4$ per group). Data are representative of 3 independent experiments.

vided by H. von Boehmer (Harvard University, Boston, Massachusetts, USA) (27). The DO.11 TCROVA transgenic mice in BALB/c background that express a TCR recognizing an I-A $\mathrm{A}^{\mathrm{d}}$-restricted OVA epitope ( ${ }^{323}$ ISQAVHAAHAEINEAGR ${ }^{339}$ ) were purchased from The Jackson Laboratory (28).

TCR-HA and TCR-OVA transgenic mice were backcrossed to the B10.D2 background and then inter-

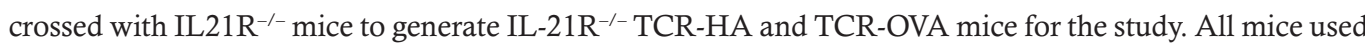
for experiments were between 8 and 10 weeks of age.

Viruses and in vivo immunization. Recombinant VV-HA and recombinant E1-deleted Ad-HA were previously described (29). Recombinant VV-OVA was provided by Jonathan Yewdell at NIH. Recombinant adenovirus encoding OVA (Ad-lacZ) was generated by homologous recombination in E. coli as described previously (30). VV-HA or VV-OVA was grown in TK-143B cells, purified by sucrose banding, and titer was determined by plaque-forming assay on TK-143B cells. Mice were infected with $5 \times 10^{6}$ pfu i.p. Ad-HA or Ad-OVA was grown in 293 cells (ATCC), purified by 2 rounds of $\mathrm{CsCl}$ density centrifugation, and desalted by gel filtration through a Sephadex G-25 column (PD-10 column, Amersham Bioscience). The titer was determined by plaque-forming assay on 293 cells. Mice were infected with $2 \times 10^{9}$ pfu i.p.

Adoptive transfer of T cells. Naive clonotypic HA-specific (or OVA-specific) CD4 T cells were prepared from WT and IL-21R ${ }^{-/} 6.5$ (or DO11) TCR transgenic mice. Briefly, single-cell suspensions were prepared from spleen and lymph nodes and subject to FACS sorting. Sorted $\mathrm{TCR}^{+} \mathrm{CD} 4^{+} \mathrm{T}$ cells were injected into recipient mice via tail vein in $200 \mu \mathrm{HBSS}$. In some experiments, cells were labeled with CFSE before transfer.

Antibodies and flow cytometry. All mAbs were purchased from BD Biosciences unless otherwise specified, including PE-Cy5-conjugated anti-CD4, anti-Thy1.2, and streptavidin; FITC-conjugated anti-CD8, anti-CD44, anti-CD69, anti-Thy1.2, anti-pSTAT1, and anti-pSTAT3; PE-conjugated antiThy1.2, anti-Bcl-xL (Santa Cruz Biotechnology), annexin V, and anti-mouse IgG (SouthernBiotech); and PE-conjugated anti-pAkt, purified anti-Akt (pan), allophycocyanin-conjugated anti-IFN- $\gamma$, and purified anti-STAT1 and -STAT3 (Cell Signaling Technology). Flow cytometry data were collected by FACSCanto (BD Biosciences) and then analyzed using FACSDiva (BD Biosciences). 
Intracellular staining. To measure IFN- $\gamma$ production in $\mathrm{CD}^{+} \mathrm{T}$ cells, splenocytes were incubated with $5 \mu \mathrm{g} / \mathrm{ml}$ brefeldin A and $20 \mu \mathrm{g} / \mathrm{ml}$ influenza HA peptide $\left({ }^{110}\right.$ SFERFEIFPKE $\left.{ }^{120}\right)$ for 6 hours at $37^{\circ} \mathrm{C}$. After stained with anti-CD4 and anti-Thy1.2, cells were permeabilized with the Cytofix/Cytoperm kit (BD Biosciences), as per the manufacturer's instructions. Permeabilized cells were subsequently stained with antiIFN- $\gamma$. To study Bcl- $x_{\mathrm{L}}$, total STAT1, STAT3, pSTAT1, and pSTAT3, cells derived from in vitro culture and stimulation or from splenocytes of infected mice were first fixed with 3.7\% formaldehyde. Afterward, cells were permeabilized with $90 \%$ ethanol, blocked with 3\% FBS, and then stained with anti-CD4, anti-Thy 1.2 , and $\mathrm{Bcl}-\mathrm{x}_{\mathrm{L}}$, STAT1, STAT3, pSTAT1, or pSTAT3.

$T$ cell culture and stimulation. Polyclonal $\mathrm{CD}^{+} \mathrm{T}$ cells were selected from splenocytes by anti-CD4 microbeads selection (Miltenyi Biotec). To measure total STAT1 and STAT3 and their phosphorylation in vitro, $2 \times 10^{5}$ naive T cells were cultured in RPMI 1640 supplemented with 10\% FBS, 2 mM L-glutamine, $100 \mathrm{IU} / \mathrm{ml}$ penicillin, $100 \mathrm{IU} / \mathrm{ml}$ streptomycin, and $50 \mu \mathrm{M}$ 2-ME, with or without IL-21 (10 ng/ml) for 1 hour and then were subjected to intracellular staining.

Retroviral transduction of T cells. The pMSCV vectors that encode dominant-negative Akt (pMSCV-dn-Akt) and constitutive Akt (pMSCV-constitutive-Akt) were gifts from Z. Songyang (Baylor College of Medicine, Houston, Texas, USA) (31), whereas the one encoding dominant-negative STAT3 (pMSCV-dn-STAT3) was from D. Link (Washington University, St. Louis, Missouri, USA) (32). After a plasmid DNA transfection with $\mathrm{CaCl}_{2}$, retroviruses were grown in BOSC packaging cells in DMEM medium (Cellgro) with 10\% FBS, penicillin, streptomycin, and glutamine. Three days after transfection, the supernatant of cell culture was collected to infect CD4 T cells that had been cultured in the presence of IL-2 (100 U/ml), PMA (40 ng/ml), and ionomycin $(500 \mathrm{ng} / \mathrm{ml})$ for 24 hours. Cells were centrifuged at 1,300 $\mathrm{g}$ for 2 hours in the presence of $8 \mathrm{~g} /$ $\mathrm{ml}$ polybrene. Three days after the transduction, GFP-positive cells were used for analysis.

Statistics. All statistical analyses were performed with Graph Prism 6 software. Results are expressed as mean \pm SEM. Significance between experimental groups was determined by a 2-tailed Student's $t$ test. A $P$ value of less than 0.05 was considered to be statistically significant for all results.

Study approval. All animal procedures were approved by the Institutional Animal Care and Use Committee at Duke University, Durham, North Carolina, USA.

\section{Author contributions}

Yuqing Yuan designed and performed the study, collected and analyzed data, and prepared the paper. Xiaopei Huang and Yiping Yang designed research, analyzed data, and wrote the paper.

\section{Acknowledgments}

This work was supported by NIH grants CA136934, CA186973, and CA193167 (to Y. Yang).

Address correspondence to: Yiping Yang or Xiaopei Huang, Department of Medicine, Duke University Medical Center, Box 103005, Durham, North Carolina 27710, USA. Phone: 919.668.0932; E-mail: yang0029@mc.duke.edu (Y. Yang). Phone: 919.668.0979; E-mail: huang027@mc.duke.edu (X. Huang).

1. Parrish-Novak J, et al. Interleukin 21 and its receptor are involved in NK cell expansion and regulation of lymphocyte function. Nature. 2000;408(6808):57-63.

2. Coquet JM, et al. IL-21 is produced by NKT cells and modulates NKT cell activation and cytokine production. J Immunol. 2007;178(5):2827-2834.

3. Leonard WJ, Spolski R. Interleukin-21: a modulator of lymphoid proliferation, apoptosis and differentiation. Nat Rev Immunol. 2005;5(9):688-698.

4. Kasaian MT, et al. IL-21 limits NK cell responses and promotes antigen-specific T cell activation: a mediator of the transition from innate to adaptive immunity. Immunity. 2002;16(4):559-569.

5. Ozaki K, et al. A critical role for IL-21 in regulating immunoglobulin production. Science. 2002;298(5598):1630-1634.

6. Ozaki K, et al. Regulation of B cell differentiation and plasma cell generation by IL-21, a novel inducer of Blimp-1 and Bcl-6. $J$ Immunol. 2004;173(9):5361-5371.

7. Zeng R, et al. Synergy of IL-21 and IL-15 in regulating CD8+ T cell expansion and function. J Exp Med. 2005;201(1):139-148.

8. Fazilleau N, Mark L, McHeyzer-Williams LJ, McHeyzer-Williams MG. Follicular helper T cells: lineage and location. Immunity. 2009;30(3):324-335.

9. Korn T, et al. IL-21 initiates an alternative pathway to induce proinflammatory T(H)17 cells. Nature. 2007;448(7152):484-487.

10. Nurieva R, et al. Essential autocrine regulation by IL-21 in the generation of inflammatory T cells. Nature. 2007;448(7152):480-483.

11. Elsaesser H, Sauer K, Brooks DG. IL-21 is required to control chronic viral infection. Science. 2009;324(5934):1569-1572.

12. Yi JS, Du M, Zajac AJ. A vital role for interleukin-21 in the control of a chronic viral infection. Science. 2009;324(5934):1572-1576. 
13. Fröhlich A, et al. IL-21R on T cells is critical for sustained functionality and control of chronic viral infection. Science. 2009;324(5934):1576-1580.

14. Novy P, Huang X, Leonard WJ, Yang Y. Intrinsic IL-21 signaling is critical for CD8 T cell survival and memory formation in response to vaccinia viral infection. J Immunol. 2011;186(5):2729-2738.

15. Yi JS, Ingram JT, Zajac AJ. IL-21 deficiency influences CD8 T cell quality and recall responses following an acute viral infection. J Immunol. 2010;185(8):4835-4845.

16. Cui W, Liu Y, Weinstein JS, Craft J, Kaech SM. An interleukin-21-interleukin-10-STAT3 pathway is critical for functional maturation of memory CD8+ T cells. Immunity. 2011;35(5):792-805

17. Ostiguy V, Allard EL, Marquis M, Leignadier J, Labrecque N. IL-21 promotes T lymphocyte survival by activating the phosphatidylinositol-3 kinase signaling cascade. J Leukoc Biol. 2007;82(3):645-656.

18. Harty JT, Badovinac VP. Shaping and reshaping CD8+ T-cell memory. Nat Rev Immunol. 2008;8(2):107-119.

19. Lanzavecchia A, Sallusto F. Progressive differentiation and selection of the fittest in the immune response. Nat Rev Immunol. 2002;2(12):982-987

20. Quigley M, Huang X, Yang Y. Extent of stimulation controls the formation of memory CD8 T cells. J Immunol. 2007;179(9):5768-5777.

21. Teixeiro E, et al. Different T cell receptor signals determine CD8+ memory versus effector development. Science. 2009;323(5913):502-505.

22. Song J, Salek-Ardakani S, Rogers PR, Cheng M, Van Parijs L, Croft M. The costimulation-regulated duration of PKB activation controls T cell longevity. Nat Immunol. 2004;5(2):150-158.

23. Schluns KS, Kieper WC, Jameson SC, Lefrançois L. Interleukin-7 mediates the homeostasis of naïve and memory CD8 T cells in vivo. Nat Immunol. 2000;1(5):426-432.

24. Zhang X, Sun S, Hwang I, Tough DF, Sprent J. Potent and selective stimulation of memory-phenotype CD8+ T cells in vivo by IL-15. Immunity. 1998;8(5):591-599.

25. Zeng R, Spolski R, Casas E, Zhu W, Levy DE, Leonard WJ. The molecular basis of IL-21-mediated proliferation. Blood. 2007;109(10):4135-4142.

26. Quigley M, Huang X, Yang Y. STAT1 signaling in CD8 T cells is required for their clonal expansion and memory formation following viral infection in vivo. J Immunol. 2008;180(4):2158-2164.

27. Kirberg J, Baron A, Jakob S, Rolink A, Karjalainen K, von Boehmer H. Thymic selection of CD8+ single positive cells with a class II major histocompatibility complex-restricted receptor. J Exp Med. 1994;180(1):25-34.

28. Murphy KM, Heimberger AB, Loh DY. Induction by antigen of intrathymic apoptosis of CD4+CD8+TCRlo thymocytes in vivo. Science. 1990;250(4988):1720-1723.

29. Yang Y, Huang CT, Huang X, Pardoll DM. Persistent Toll-like receptor signals are required for reversal of regulatory T cell-mediated CD8 tolerance. Nat Immunol. 2004;5(5):508-515.

30. He TC, Zhou S, da Costa LT, Yu J, Kinzler KW, Vogelstein B. A simplified system for generating recombinant adenoviruses. Proc Natl Acad Sci USA. 1998;95(5):2509-2514.

31. Songyang Z, Baltimore D, Cantley LC, Kaplan DR, Franke TF. Interleukin 3-dependent survival by the Akt protein kinase. Proc Natl Acad Sci USA. 1997;94(21):11345-11350.

32. McLemore ML, et al. STAT-3 activation is required for normal G-CSF-dependent proliferation and granulocytic differentiation Immunity. 2001;14(2):193-204. 